\title{
Iris Recognition: An Analysis of the Aliasing Problem in the Iris Normalization Stage
}

\author{
Hugo Proença and Luís A. Alexandre \\ Dep. Informatics, Universidade da Beira Interior \\ IT - Networks and Multimedia Group, Covilhã \\ R. Marquês D’Ávila e Bolama, 6200-001, Covilhã, Portugal \\ \{hugomcp, lfbaa\}@di.ubi.pt
}

\begin{abstract}
Iris recognition has been increasingly used with very satisfactory results. Presently, the challenge consists in unconstrain the image capturing conditions and enable its application to domains where the subjects' cooperation is not expectable (e.g. criminal/terrorist seek, missing children). In this type of use, due to variations in the image capturing distance and in the lighting conditions that determine the size of the subjects' pupil, the area correspondent to the iris in the captured images will be highly varying too. In order to compensate this variation, common iris recognition proposals translate the segmented iris image to a double dimensionless pseudo-polar coordinate system, in a process known as the normalization stage, which can be regarded as a sampling of the original data with the inherent possibility of aliasing. In this paper we analyze the relationship between the size of the captured iris image and the overall recognition's accuracy. Further, we identify the threshold for the sampling rate of the iris normalization process above which the error rates significantly increase.
\end{abstract}

Keywords: iris normalization, aliasing, iris recognition, biometrics.

\section{Introduction}

In 1987, L. Flom and A. Safir [5] estimated at 1 in $10^{72}$ the probability for the existence of two similar irises and concluded about the stability of iris morphology over human lifetime. Since then, the use of the iris as biometric measure has been increasingly encouraged by both government and private entities. Iris is commonly recognized as one of the most reliable biometric signals: it has a random morphogenesis and apparently no genetic penetrance.

From our viewpoint, the present challenge consists in achieve accurate iris recognition in less constrained image capture environments, either under natural luminosity, from different image capturing distances and without users' cooperation.

In order to achieve invariance to the varying size of the pupil and to the distance and angle of the image capturing framework, common iris recognition proposals apply a normalization process to the segmented iris. The translation to a double dimensionless pseudo-polar coordinate system with fixed dimensions can be regarded as a sampling process, with the inherent possibility of aliasing that deteriorates the recognition's accuracy. 
Given a power spectrum (a plot of power versus frequency), aliasing is a false translation of power falling in some frequency range $\left(-f_{c}, f_{c}\right)$ outside the range. It can be caused by discrete sampling below the Nyquist frequency and causes that different signals could become indistinguishable when sampled. When this happens, the original signal cannot be uniquely reconstructed from the sampled signal.

In this paper we analyze the relationship between the size of the captured iris images and the iris recognition's accuracy, regarding the probability of aliasing in the normalization stage.

We selected two highly dissimilar iris image data sets (UBIRIS [12] and UPOL [4]) and analyzed the results obtained by the classical Daugman's recognition method [2], when varying the size of the captured iris images.

It will be shown that when the area correspondent to the iris in the captured image is below $30 \%$ of the size of the normalized one, it occurs a large deterioration in the recognition accuracy, specially due to a substantial increase of the false rejections.

The remainder of this paper is organized as follows: section 2 briefly summarizes the most cited iris recognition methods. A detailed description of two common iris normalization proposals is given in section 2.2. Section 3 reports the experiments and results and, finally, section 4 concludes this paper.

\section{Iris Recognition}

\subsection{Overview}

In spite of the specificities from distinct proposals, typical iris recognition systems share the common structure illustrated by figure 1 .

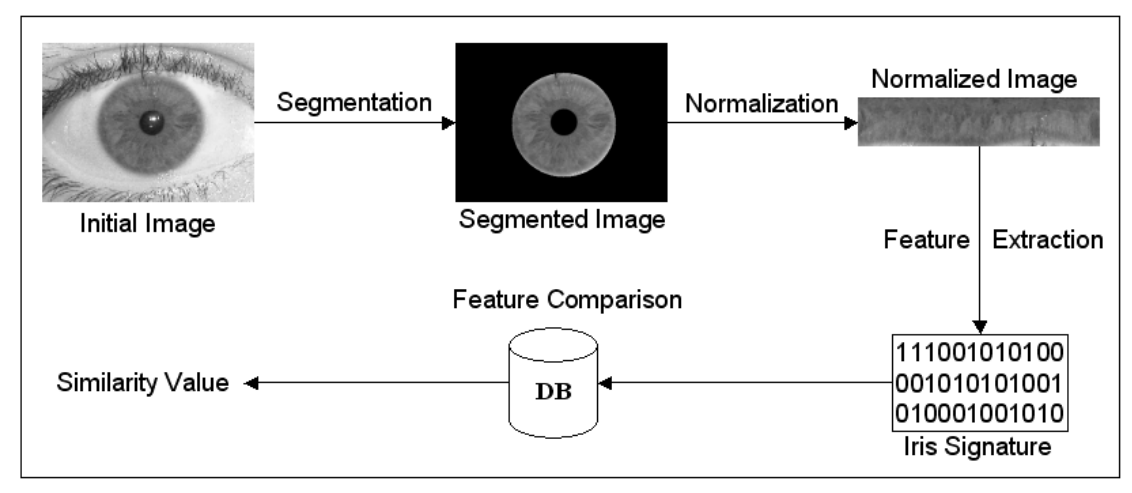

Fig. 1. Typical stages of the iris recognition systems

The initial stage deals with iris segmentation. This consists in localize the iris inner (pupillary) and outer (scleric) borders. There are two major strategies for iris segmentation: to use a rigid or deformable template of the iris or its boundary. In most cases, 
the boundary approach is very similar to the proposed by Wildes [16]: it begins by the construction of an edges map followed by the application of some geometric form fitting algorithm. Authors of [13] used this strategy together with a clustering process to increase the accuracy in noisy environments. The template-based strategies usually involve the maximization of some equation, as proposed by Daugman [2] and Roche et. all [10].

In order to compensate the varying size of the captured iris it is common to translate the segmented iris region, represented in the cartesian coordinate system, to a fixed length and dimensionless polar coordinate system. This is usually accomplished through a method similar to the Daugman's Rubber Sheet [2].

The next stage is the feature extraction. From this viewpoint, iris recognition approaches can be classified into three major categories: phase-based methods (e.g. [2]), zero crossing methods (e.g. [1] and [10]) and texture analysis based methods (e.g [16], [7] and [9]).

Daugman [2] uses multiscale quadrature wavelets to extract texture phase information and obtain an iris signature with 2048 binary components. To characterize the texture from the iris, Boles and Boashash [1] computed the zero-crossing representation of a 1D wavelet at different resolutions of concentric circles. Wildes [16] proposes the characterization of the iris texture through a Laplacian pyramid with 4 different levels (scales). This pyramid is further used to compute the normalized correlation between images and conclude about their similarity. One of the most typical approaches constitutes the proposal of [8], characterizing the iris texture through its decomposition by the dyadic wavelet transform, either using Haar, Daubechies or other mother wavelets. This approach was found with minor variants in several other works (e.g. [14]).

In the final stage it is made a comparison between iris signatures, producing a numeric dissimilarity value. If this value is higher than a threshold, the system outputs a "non-match", meaning that each signature belongs to different irises. Otherwise, the system outputs a "match", meaning that both signatures were extracted from images of the same iris. This stage is highly constrained by the feature extraction method. When the produced iris signature is binary, it is common to apply the Hamming distance metric. Otherwise, different metrics like the Euclidean, Weighted Euclidean or methods based on signal correlation (e.g. [16]) can be applied.

\subsection{Iris Normalization Methods}

Robust representations for pattern recognition must be invariant to changes in the size, position and orientation of the patterns. In the iris biometric compass, this means that a representation of the iris data invariant to changes in the distance between the eye and the capturing device, in the camera optical magnification factor and in the iris orientation, caused by torsional eye rotation and camera angles, must be accomplished. As described in [3], the invariance to all of these factors can be achieved by the translation of the captured data to a double dimensionless pseudo-polar coordinate system. Figure 2 illustrates the translation process, that is based in a polar $(\theta)$ and radial $(r)$ variables.

The rubber sheet is a linear model that assigns to each pixel of the iris, regardless its size and pupillary dilation, a pair of real coordinates $(r, \theta)$, where $r$ is on the unit 


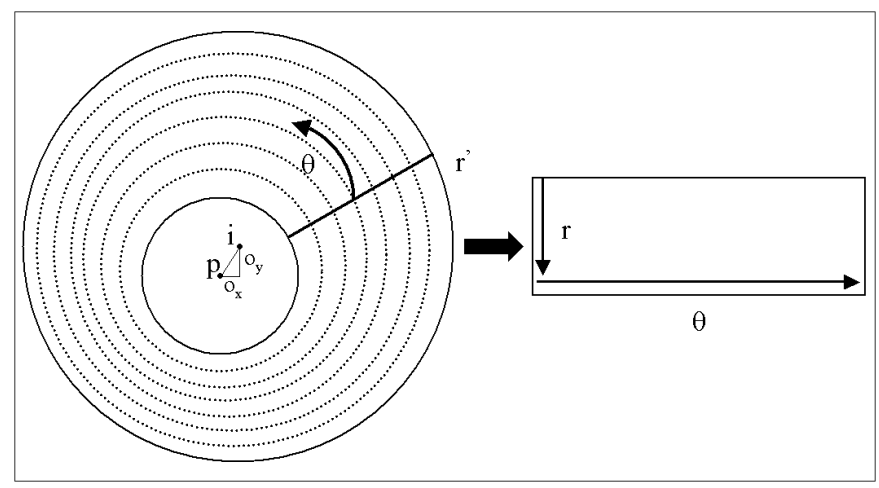

Fig. 2. Normalization of the iris image through the "Daugman rubber sheet" model [3]. "i" and "p" represent respectively the center of the iris and of the pupil. $\left(o_{x}, o_{y}\right)$ is the difference between both centers. The normalization is anti-clockwise processed, extracting a fixed number of pixels from circumferences with successive radius values, into the normalized rectangular image with fixed dimensions.

interval $[0,1]$ and $\theta$ is an angle in $[0,2 \pi]$. The remapping of the iris image $I(x, y)$ from raw cartesian coordinates $(x, y)$ to the dimensionless non concentric polar coordinate system $(r, \theta)$ can be represented as:

$$
I(x(r, \theta), y(r, \theta)) \rightarrow I(r, \theta)
$$

where $x(r, \theta)$ and $y(r, \theta)$ are defined as linear combinations of both the set of pupillary boundary points $\left(x_{p}(\theta), y_{p}(\theta)\right)$ and the set of limbus boundary points along the outer perimeter of the iris $\left(x_{s}(\theta), y_{s}(\theta)\right)$ bordering the sclera:

$$
\left\{\begin{array}{l}
x(r, \theta)=(1-r) * x_{p}(\theta)+r * x_{s}(\theta) \\
y(r, \theta)=(1-r) * y_{p}(\theta)+r * y_{s}(\theta)
\end{array}\right.
$$

Authors of [17] proposed a slightly different iris normalization model, which combines linear and non-linear methods to unwrap the iris region. They start by performing a non-linear transformation of all iris patterns to a reference annular zone with a predefined ratio of the radii of inner and outer boundaries of the iris. Further, this reference annular zone is linearly unwrapped to a fix-sized rectangle block for subsequence processing.

Both normalization processes can be regarded as point sampling operators, defined by:

$$
s(I)=\left(I\left(t_{1}\right), I\left(t_{2}\right), \ldots, I\left(t_{n}\right)\right), t_{i}=\frac{i}{n}, i=1, \ldots, n
$$

where $I$ is the original signal (image). 


\subsection{Aliasing}

Let $I_{1}$ and $I_{2}$ be two iris images similar to the "Initial Image" of figure 1. Also, let $A(I)$ denote the area correspondent to the iris in image $I$ ("Segmented Image" of figure 1). In the normalization stage $(s)$, as in any other point sampling process, aliasing can occur in two distinct forms:

- $I_{1}$ and $I_{2}$ are very dissimilar and $s\left(I_{1}\right)$ and $s\left(I_{2}\right)$ are highly similar. In the iris biometric compass, this will increase the false accept rate (FAR).

- $I_{1}$ and $I_{2}$ are very similar and $s\left(I_{1}\right)$ and $s\left(I_{2}\right)$ are are highly dissimilar, increasing the false rejection rate (FRR).

Commonly, the normalized iris images have fixed dimensions of $512 \times 64$ pixels, respectively in the angular and radial directions, thus $A(s(I))=32768$ pixels. The sampling rate $r$ of the normalization process $s$ can be given by:

$$
r=\frac{A(s(I))}{A(I)}=\frac{32768}{A(I))}
$$

where $I$ is the captured iris image.

In our experiments, we varied the size of the captured iris image $(A(I))$ and analyzed its influence in the overall accuracy of iris recognition. It will be shown that when $r>4$ there is a strong increase of the recognition error rates, induced by the aliasing occurred in the normalization process.

\section{Experiments}

In the experiments, we implemented the method described by Daugman [2].

\subsection{Daugman's Method}

As described in [2], the Daugman's recognition method is composed by the following stages:

- Iris segmentation. We implemented the integrodifferential operator proposed by the author to find both the inner and outer iris borders, given by:

$$
\max _{r, x_{0}, y_{0}}\left|G_{\sigma}(r) * \frac{\delta}{\delta r} \oint_{r, x_{0}, y_{0}} \frac{I(x, y)}{2 \pi r} d s\right|
$$

This operator searches over the image domain $(x, y)$ for the maximum in the blurred partial derivative (by a Gaussian kernel $G_{\sigma}$ with respect to increasing radius $r$, of the normalized contour integral of $I(x, y)$ along a circular arc $d s$ of radius $r$ and center coordinates $\left(x_{0}, y_{0}\right)$.

- Normalization. To compensate the variations in the size of the pupil, we translated the images to dimensionless polar coordinate system through a process known as the "Daugman Rubber Sheet" [2] that is described in section 2.2. 
- Feature Extraction. The iris data encoding was accomplished through the use of two dimensional Gabor filters. These spatial filters have the form:

$$
G(x, y)=e^{-\pi\left[\left(x-x_{0}\right)^{2} / \alpha^{2}+\left(y-y_{0}\right)^{2} \beta^{2}\right]} \cdot e^{-2 \pi i\left[u_{0}\left(x-x_{0}\right)+v_{0}\left(y-y_{0}\right)\right]}
$$

where $\left(x_{0}, y_{0}\right)$ defines the position in the image, $(\alpha, \beta)$ is the filter width and length and $\left(u_{0}, v_{0}\right)$ specify the modulation, with spatial frequency $w_{0}=\sqrt{u_{0}^{2}+v_{0}^{2}}$ and direction $\theta_{0}=\arctan \left(v_{0} / u_{0}\right)$.

The real parts of the 2-D Gabor filters are truncated to be zero volume and achieve illumination invariance. For each resulting bit the sign of the real and imaginary parts from quadrature image projections are analyzed and through quantization assigned binary values: 1 and 0 respectively for positive and negative projection values.

- Feature Comparison. The binarization process in the feature extraction allows the use of the Hamming distance as similarity measure for iris signatures. Given two binary sets with $N$ bits: $A=\left\{a_{1}, \ldots, a_{N}\right\}$ and $B=\left\{b_{1}, \ldots, b_{N}\right\}$, the Hamming distance is:

$$
H D(A, B)=\frac{1}{N} * \sum_{i=1}^{N} a_{i} \otimes b_{i}
$$

where $a \otimes b$ is the logical "XOR" operation. Thus, for two completely equal and different signatures, the value of the Hamming distance will be respectively 0 and 1.

\subsection{Data Sets}

There are presently 5 public and free available iris image databases for biometric purposes: CASIA [6], MMU [11], BATH [15], UPOL [4] and UBIRIS [12].

CASIA database is by far the most widely used for iris biometric purposes. However, its images incorporate few types of noise, almost exclusively related with eyelid and eyelash obstruction, as the images from MMU and BATH databases. UPOL images were captured with an optometric framework, obtaining optimal images with extremely similar characteristics. Oppositely, UBIRIS database was builded with the objective of simulate non-cooperative image capturing. This fact explains the higher heterogeneity of its images.

Based on this, we chosen two data sets from the databases with most opposite characteristics: $U B I R I S$ and $U P O L$, in order to enable the analysis of the recognition's accuracy in high quality images ( $U P O L$ ) and in lower quality (highly noisy) images $(U B I R I S)$. These types of images correspond respectively to the image capturing with and without users' cooperation.

We selected 130 images from each database, belonging to 13 different subjects. Table 1 contains information about the images of each data set. The first column identifies the data set, the second specifies the dimensions of the images. The third and forth columns contain respectively the average radius of the pupil and the iris. Finally, the fifth and sixth columns contain the average area of the iris ring and the average 
sampling rate (4) of the normalization processes for the images of the data set. As can be seen in figure 3, although smaller than the UBIRIS images, the iris region in the $U P O L$ images is much larger, corresponding to smaller sampling rates of the normalization process.

In order to avoid that segmentation errors corrupt the obtained results, we manually verified that the segmentation algorithm accurately segmented all the images from both data sets.

The simulation of the different sizes of the captured iris images was accomplished through bi-cubic resizing of the original images of the data sets. Each image was resized from $100 \%$ to $10 \%$ of its original size.

\begin{tabular}{|l|c|c|c|c|c|}
\hline Data set & Image size & Pupil radius & Iris radius & Iris area & Sampling rate \\
\hline$U B I R I S$ & $800 \times 600$ & 51 & 185 & 99347 & 0.3298 \\
\hline$U P O L$ & $768 \times 576$ & 69 & 271 & 215758 & 0.1518 \\
\hline
\end{tabular}

Table 1. Characteristics of the data sets used in our experiments.

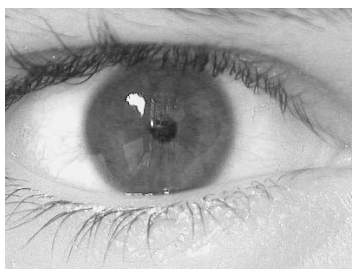

(a) Iris image from the $U$ B I RIS database

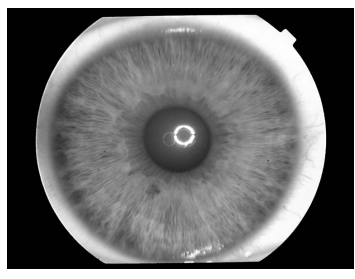

(b) Iris image from the $U P O L$ database.

Fig. 3. Examples of images from the data sets used in the experiments.

\subsection{Results}

According to (4), figure 4 contains the average sampling rate of the normalization processes in the images from UBIRIS (figure 4a) and UPOL (figure 4b) data sets as the dimension of the captured iris images varies. The horizontal axis specifies the size of the captured iris images proportionally to the size of the images described in table 1 (values are percent). The vertical axis contains the average sampling rate $(r)$ of the normalization processes of these images.

Figure 5 contains four measures of the recognition's accuracy. One again, the horizontal axis denotes the size of the used images in proportion (percentage) with the size of the images described in table 1.

Figure 5a contains the values for a t-test given by: 


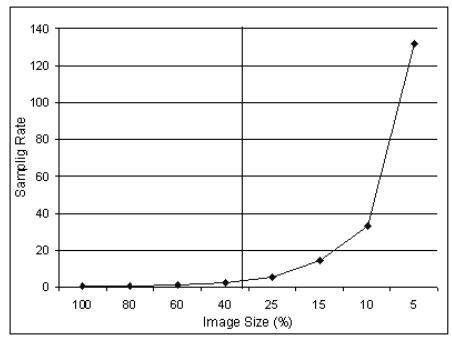

(a) Average sampling rate $(r)$ from the images of U BI RIS data set.

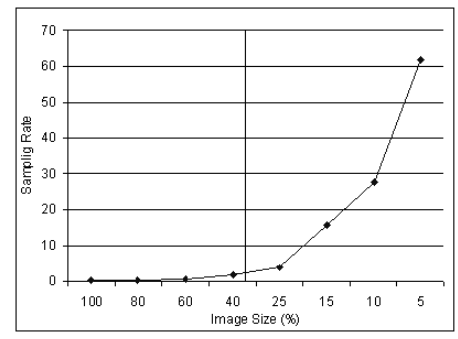

(b) Average sampling rate ( $r$ ) from the images of $U P O L$ data set.

Fig. 4. Average sampling rate $(r)$ of the normalization process versus the size of the captured iris images, given by the proportion with the images' size specified in table 1 . The vertical line identifies the threshold from which we observed that the recognition's accuracy significantly decreases.

$$
\tau=\frac{\mu_{E}-\mu_{I}}{\sqrt{\frac{\sigma_{I}^{2}}{N_{I}}+\frac{\sigma_{E}^{2}}{N_{E}}}}
$$

where symbols $\mu_{I}$ and $\mu_{E}$ respectively indicate the means of the intra- (images from the same iris) and inter-class (images from different irises) comparisons. $\sigma_{I}$ and $\sigma_{E}$ indicate the respective standard deviations and $N_{I}$ and $N_{E}$ the total intra- and interclass comparisons.

Figure $5 \mathrm{~b}$ contains the equal error rates and figure $5 \mathrm{c}$ the percent values for the areas under the receiver operating curves (ROC). Finally, figure $5 \mathrm{~d}$ contains the values of the false rejections when the false acceptances are minimized.

We observed that the separability between the intra- and inter-class comparisons, given by equation (5), remained with similar values until the iris area in the captured image is below $40 \%$ of the normalized image one, either in the UBIRIS and in the $U P O L$ data sets. Moreover, when the area of the original data is below $30 \%$ of the normalized one there is a significant decrease in the separability between the intraand inter-class comparisons, corresponding to sampling rates (4) higher than 5. Above this value we observed a significant increment of the error rates, specially the false rejections, allowing the conclusion of aliasing in the iris normalization stage.

\section{Conclusions}

In this paper we analyzed the influence of the sampling rate of the iris normalization stage in the overall accuracy of iris recognition.

We observed no significant degradation in the accuracy when the sampling rates are lower than 5. For higher sampling rates (correspondent to original images with iris area below $30 \%$ of the normalized one), the error rates significantly increase. 


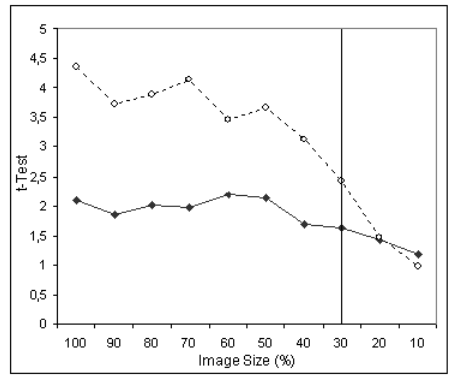

(a) $t$-Test values.

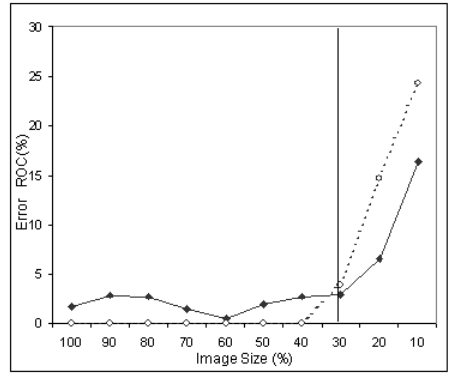

(c) Error Area Under the ROC Curve.

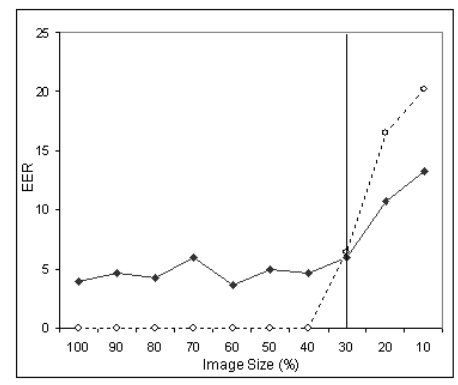

(b) Equal Error Rate (EER).

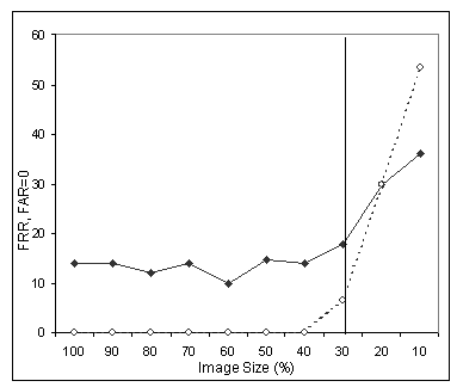

(d) FRR with FAR=0.

Fig. 5. Iris recognition's accuracy regarding the size of the images presented to the segmentation algorithm and used as basis in the iris normalization (sampling) stage. The solid line represents the results obtained in the $U B I R I S$ data set and the dashed line represents the results obtained in the $U P O L$ data set. The vertical line corresponds to the identified threshold of $30 \%$, from which the error rates significantly increase.

This fact indicates a strong probability of aliasing when iris images are captured at a distance. From our viewpoint, the increase of the error rates, specially the false rejections, requires alternate sampling/normalization processes more tolerant to highest variations in the size of the captured iris images.

Moreover, we stress that the observed deterioration in the recognition's accuracy is independent of the amount of noise that the iris region contain, since the values obtained for the minimum demandable sampling rates in the $U B I R I S$ (noisy images) and in the $U P O L$ (high quality images) data sets were approximately equal.

\section{References}

1. W. W. Boles and B. Boashash. A human identification technique using images of the iris and wavelet transform. IEEE Transactions on Signal Processing, vol. 46, no. 4, pages 11851188, April 1998. 
2. John G. Daugman. High confidence visual recognition of persons by a test of statistical independence. IEEE Transactions on Pattern Analysis and Machine Intelligence, Vol. 25, No. 11, pages 1148-1161, November 1993.

3. John G. Daugman. How iris recognition works. IEEE Transactions on Circuits and Systems for Video Technology, Vol. 14, No. 1, pages 21-30, January 2004.

4. Michal Dobes and Libor Machala. UPOL iris image database, 2004. http://phoenix.inf.upol.cz/iris/

5. L. Flom and A. Safir. Iris recognition system, 1987. U.S. Patent 4641394.

6. Institute of Automation, Chinese Academy of Sciences. CASIA iris image database, 2004. http: //www.sinobiometrics.com

7. Jaemin Kim, Seongwon Cho, and Jinsu Choi. Iris recognition using wavelet features. Kluwer Academic Publishers, Journal of VLSI Signal Processing, no. 38, pages 147-256, November 2004.

8. Shinyoung Lim, Kwanyong Lee, Okhwan Byeon, and Taiyun Kim. Efficient iris recognition through the improvement of feature vector and classifier. ETRI Journal, vol. 23, no. 2, pages 61-70, June 2001.

9. Li Ma, Yunhong Wang, and Tieniu Tan. Iris recognition using circular symmetric filters. In Proceedings of the 25th International Conference on Pattern Recognition (ICPRO2), vol. 2, pages 414-417, 2002.

10. D. Martin-Roche, C. Sanchez-Avila, and R.Sanchez-Reillo. Iris recognition for biometric identification using dyadic wavelet transform zero-crossing. IEEE Aerospace and Electronic Systems Magazine, Mag. 17, No. 10, pages 3-6, 2002.

11. Multimedia University. MMU iris image database, 2004. http: //pesona.mmu.edu.my/ccteo

12. Hugo Proença and Luís A. Alexandre. UBIRIS: A noisy iris image database. In 13th International Conference on Image Analysis and Processing (ICIAP2005), pages 970-977, September 2005. http://iris.di.ubi.pt

13. Hugo Proença and Luís A. Alexandre. Iris segmentation methodology for non-cooperative iris recognition. IEE Proc. Vision, Image \& Signal Processing, vol. 153, issue 2, pages 199 205, 2006.

14. C. Sanchez-Avila and D. Martin-Roche. Iris-based biometric recognition using dyadic wavelet transform. IEEE Aerospace and Electronic Systems Magazine, pages 3-6, 2002.

15. University of Bath. University of Bath iris image database, 2004. www.bath.ac.uk/elec-eng/pages/sipg/

16. Richard P. Wildes. Iris recognition: an emerging biometric technology. In Proceedings of the IEEE, vol. 85, no.9, pages 1348-1363, U.S.A., September 1997.

17. Xiaoyan Yuan and Pengfei Shi. A non-linear normalization model for iris recognition. In Proceedings of the International Workshop on Biometric Recognition Systems IWBRS 2005, pages 135-142, China, 2005. 